\title{
X-RAY BINARIES IN EARLY TYPE GALAXIES AND THE GLOBULAR CLUSTER CONNECTION
}

\author{
T. J. Maccarone, ${ }^{1}$ A. Kundu, ${ }^{2}$ S. E. Zepf, ${ }^{2}$ and T. H. Puzia ${ }^{3}$ \\ RESUMEN
}

Presentamos un resumen de las observaciones claves de fuentes de rayos X realizadas recientemente en galaxiats de tipos tempranos. Típicamente, alrededor de la mitad de las binarias de rayus X en galaxias de tipos tempranos se encuentra en cúmulos globulares, preferentemente en los que son ricos en metiales. sin haber indicios de que la edad del cúmulo sea un parámetro importante. Estos resultados presentan desafíos teóricos.

\section{ABSTRACT}

We summarize the key observations made in recent observations of X-ray sources in early-type galaxies. Typically about half of the X-ray binaries in early-type galaxies are in globular clusters, they are preferentially found in metal rich globular clusters, and there is no indication that cluster age is an important parameter. Theoretical challenges are presented by these results.

\section{Key Words: GALAXIES: INDIVIDUAL (NGC 3115,NGC 4365, NGC 4472) — GALAXIES: STAR CLUSTERS - STARS: MASS LOSS - X-RAYS: BINARIES}

\section{GENERAL}

Before Chandra, there was debate about the source of hard X-ray emission in elliptical galaxies. Correlations between the hard X-ray and optical luminosities suggested that the emission probably had an X-ray binary origin (Trinchieri \& Fabbiano 1985), but other possibilites were still suggested. Early Chandra observations resolved these X-ray binaries out, and found that typically about half the X-ray binaries were associated with globular clusters (Angelini, Loewenstein \& Mushotzky 2001; Sarazin, Irwin \& Bregman 2001; Kundu, Maccarone \& Zepf 2002).

\section{WHICH GLOBULAR CLUSTERS HAVE X-RAY BINARIES?}

Studies of NGC 4472 show that two properties of globular clusters have a significant impact on the probability it will host an X-ray binary - cluster luminosity, and cluster color (Kundu, Maccarone \& Zepf 2002). The luminosity correlation is consistent with a constant number of X-ray binaries per unit stellar mass. The color correlation indicates that the metal rich clusters are about 3 times more likely to have an $\mathrm{X}$-ray source than the metal poor clusters, although since the metal rich clusters may be younger than the metal poor clusters it is not clear that metallicity is

\footnotetext{
${ }^{1}$ Astronomical Institute "Anton Pannekoek", University of Amsterdam, Amsterdam, The Netherlands.

${ }^{2}$ Deparment of Physics and Astronomy, Michigan State University, East Lansing, MI, USA.

${ }^{3}$ Space Telescope Science Institute, Baltimore, MD, I SA.
}

truly the driving parameter. Subsequent studies of NGC 4365 and NGC 3115 indicate that the cluster age is at most a second order parameter, and that the metallicity effect will be seen even in galaxies with coeval metal rich and metal poor clusters (Kundu et al. 2003).

\section{WHICH X-RAY BINARIES ARE IN CLUSTERS?}

The X-ray luminosity functions and spatial distributions for the cluster soure's and the noncluster sources are consistent with one another in NGC 4472. This indicates that the field sources and cluster sources may have a common origin and that a large fraction of field sources may have been ejected from clusters but there is not yet a large enough dat a sample to make strong claims (Marcarone, Kundu $\&$ Zepf 2003). The sources in metal rich clusters seem to have softer low (ne'rgy X-ray spectra than the source in metal poor clusters (Irwin \& Bregman 1999; Maccarone, Kundu d Zepf 2003).

\section{HOW ARE THE FIELD SOURCES FOR\IED?}

The field X-ray binaries cannot be steadily accreting at their observed luminosities for longer than a few hundred million years, or the mass supply from the donor would be exhausted. Low duty crcle transients can live considerably longer, and may help solve the problem of why X-ray binaries exist in galaxies with no recent star formation (Piro o B Bildsten 2002). Alternatively, the field sources mav have been formed in globular clusters (White. Sarazin 
\& Kulkarni 2002) and released into the field either through dynamical interactions leading to ejections, or through tidal destruction of the clusters. In NGC 4472 the spatial profiles, luminosity functions and spectral properties of the cluster and field sources are consistent with one another, suggestion that dynamical ejection from clusters in the key formation process; the spatial profiles are much more similar to the globular cluster profile than the integrated stellar light profile (Maccarone, Kundu \& Zepf 2003). Given that the cluster sources may also be transients, the key piece of cvidence is the similarity of the spatial profiles, which is not convincing on its own; upcoming $X M M$ observations will provide much stronger constraints, since they will observe sources at large radial distances where the field star and globular cluster profiles diverge most strongly.

\section{AN EXPLANATION FOR THE METALLICITY EFFECTS}

A possibile explanation for the two metallicity effects comes from irradiation induced stellar winds. $\mathrm{X}$-rays from the accreting object will be absorbed by the mass donor. Metal rich stars will dissipate the energy through line cooling more efficiently than metal poor stars, leaving metal poor stars with stronger stellar winds to dissipate the deposited energy (Iben, Tutukov \& Fedorova 1997). The lifetimes of the metal rich systems will then be longer, and hence their numbers will be larger. The stellar wind material will also act as an absorber of soft $\mathrm{X}$-rays, making hard spectra for the metal poor systems which have stronger winds than the metal rich systems (Maccarone, Kundu \& Zepf, submitted).

\section{REFERENCES}

Angelini, L., Loewenstein, M., \& Mushotzky, R. F. 2001, ApJL, 557, 35

Iben, I., Tutukov, A. V., \& Fedorova, A. V. 1997, ApJ, 486,955

Irwin, J. A. \& Bregman, J. N. 1999, ApJL, 510, 21

Kundu, A., Maccarone, T. J., \& Zepf, S. E. 2002, ApJL, 574,5

Kundu, A., Maccarone, T. J., Zepf, S. E., \& Puzia, T. H. 2003, ApJL, 589, 81

Maccarone, T. J., Kundu, A. \& Zepf, S. E., 2003, ApJ, 586,814

Piro, A. L. \& Bildsten, L. 2002, ApJL, 571, 103

Sarazin, C. L., Irwin, J. A., Bregman, J. N. 2001, ApJ, 556,533

Trinchieri, G. \& Fabbiano, G. 1985, ApJ, 296, 447

White, R. E., Sarazin, C. L., \& Kulkarni, S. R. 2002, ApJL, 571, 23

Arunav Kundu and Stephen E. Zepf: Department of Physics and Astronomy, Michigan State University, East Lansing MI 48824-2320 (akundu,zepf@ast.man.ac.uk).

Thomas J. Maccarone: Astronomical Institute "Anton Pannekoek," Universit y of Amsterdam, Kruislaan 403, Amsterdam, 1098 SJ, The Netherlands (tjm@science.uva.nl)

Thomas H. Puzia: Space Telescope Science Institute, 3700 San Martin Drive, Baltimore, MD 21218 (puzia@ usm.uni-muenchen.de 